\title{
Insufficient Cooling
}

National Cancer Institute

\section{Source}

National Cancer Institute. Insufficient Cooling. NCI Thesaurus. Code C63071.

Problem associated with the device insufficiently cooled in device active (working) or/and non-active (nonworking) state. 